\title{
High Frequency of Stop Codons in the Human Immunodeficiency Virus-1 Protease Gene Frame in Human Immunodeficiency Virus + Individuals with Below Detectable Levels of Plasma Viremia During Highly Active Antiretroviral Therapy
}

\author{
${ }^{1}$ Magdalena Czubala, ${ }^{1}$ Kabo Matlho, ${ }^{1}$ Maria Arriaga, ${ }^{2}$ Wayne B. Dyer, \\ ${ }^{1}$ Bin Wang, ${ }^{3}$ Choo Beng Chew, ${ }^{3}$ Dominic E. Dwyer and ${ }^{1}$ Nitin K. Saksena \\ ${ }^{1}$ Retroviral Genetics Division, Westmead Millennium Institute, \\ Westmead Hospital and the University of Sydney, Westmead NSW 2145, Australia \\ ${ }^{2}$ Department of Research and Development, \\ Australian Red Cross Blood Service 17 O'Riordan Street, Alexandria, NSW 2015, Australia \\ ${ }^{3}$ Department of Virology, Westmead Hospital, Westmead NSW 2145, Sydney, Australia
}

Received 2012-09-04, Revised 2012-11-11; Accepted 2012-11-14

\begin{abstract}
We performed sequence analysis of HIV-1 proviral protease gene fragment (560 base pairs) amplified from ex-vivo peripheral blood mononuclear cells of $83 \mathrm{HIV}+$ individuals with Below Detectable Levels (BDL) $(<20-40$ RNA copies/ml plasma) and detectable levels of plasma HIV viremia while on HAART. Noteworthy was the systematic presence of stop codons identified only in the BDL group and not in individuals with detectable plasma viremia $(\mathrm{p}<0.0001)$. The stop codons dominated positions 16 and 157 in the protease gene. This suggests that specific mutations in the protease gene possibly provide transitory molecular control of viral replication to below detectable levels in plasma during HAART. Thus, these mutations could potentially be exploited for long-term control of HIV.
\end{abstract}

Keywords: Human Immunodeficiency Virus (HIV), Highly Active Antiretroviral Therapy (HAART), Stop Codons, Gag Gene, Protease Inhibitors

\section{INTRODUCTION}

How antiretroviral drugs shape the architecture of HIV over time is visible at the level of emerging drug resistance mutations and viral evolution during Highly Active Antiretroviral Therapy (HAART) (Potter et al., 2003; 2004; 2006). It is unknown if mutations emerging in the presence of NRTI, NNRTI and protease inhibitors can provide complete but transitory control of HIV replication in vivo to Below Detectable Levels (BDL) of plasma HIV ( $<20-40$ copies of HIV RNA/ml plasma). Even though HIV can be suppressed by HAART to below detectable levels in plasma, low-level HIV replication continually occurs in cellular reservoirs despite HAART (Potter et al., 2003; 2004). PCR amplification of HIV from plasma cannot be achieved successfully when the virus reaches below the limit of detection $(<20-40$ copies of HIV/ml plasma), although can be detected in the Peripheral Blood Mononuclear Cells (PBMC) as integrated provirus. Therefore, we analyzed this HIV provirus in the protease gene derived from ex-vivo obtained PBMC from $\mathrm{HIV}+$ individuals who were able to achieve complete control of plasma HIV by HAART and compared them against individuals who displayed varying ranges of plasma viremia while on HAART.

Corresponding Author: Nitin K. Saksena, Retroviral Genetics Division, Westmead Millennium Institute, Westmead Hospital and the University of Sydney, Westmead NSW 2145, Australia Tel: +61 298459119 Fax: +61 298459103 
Magdalena Czubala et al. / American Journal of Infectious Diseases 8 (3) (2012) 115-122

Table 1. Details of positions of stop codons, insertions and deletions in the gag gene derived from HIV-strains from HIV patients showing complete control of viremia while on HAART and HIV patients failing HAART. The positions of the codons is compared to HIVK2007 (subtype B) as shown in Fig. 1

\begin{tabular}{|c|c|c|c|c|}
\hline Patient IDs & Corresponding patient number in the figure & STOP CODON POSITION & Insertions & Deletions \\
\hline 1 & BDL1 & 16,157 & & $124-125$ \\
\hline$\frac{1}{2}$ & BDL2 & 157 & & $124-125$ \\
\hline 3 & BDL3 3 & 13 & & $124-127$ \\
\hline 4 & BDL4 & & & $124-128$ \\
\hline $\begin{array}{l}4 \\
5\end{array}$ & $\begin{array}{l}\text { BDL5 } \\
\text { BDL } 50\end{array}$ & & & $124-128$ \\
\hline 6 & BDL6 & & & $126-128$ \\
\hline 7 & BDL7 & 157 & & \\
\hline 8 & BDL8 & $13,55,73$ & & 113 \\
\hline $\begin{array}{l}9 \\
10\end{array}$ & BDL9 & & 1 in 126 & \\
\hline $\begin{array}{l}10 \\
11\end{array}$ & BDL10 & $15,76,103$ & & \\
\hline 12 & $\begin{array}{l}\text { BDL11 } \\
\text { BDL12 }\end{array}$ & 69,74 & & $\begin{array}{l}127-128 \\
127-128\end{array}$ \\
\hline 13 & BDL13 & & 1 in 126 & \\
\hline 14 & BDL14 & $21,40,72,157$ & 1 in 126 & \\
\hline 15 & BDL15 & $21,40,12,107$ & 6 in 126 & \\
\hline 16 & BDL16 & 157 & & $127-128$ \\
\hline 17 & BDL17 & 107 & & $125-126$ \\
\hline 18 & BDL18 & & & $127-128,130$ \\
\hline 19 & BDL19 & & & $114,127-128,130$ \\
\hline 20 & BDL20 & & & $125-126$ \\
\hline 21 & BDL21 & & & $125-126$ \\
\hline 22 & BDL22 & & & $124-125$ \\
\hline 23 & BDL23 & & & $124-125$ \\
\hline 24 & BDL24 & 16 & & $124-125$ \\
\hline 25 & BDL25 & 16 & 1 in 126 & \\
\hline & BDL26 & & 17120 & $124-125$ \\
\hline 27 & BDL27 & & & $124-125$ \\
\hline 28 & BDL28 & & & $124-125$ \\
\hline 29 & BDL29 & & & $124-125$ \\
\hline 30 & BDL30 & & & $124-125$ \\
\hline 31 & BDL31 & 28 & & $124-125$ \\
\hline 32 & BDL32 & 20 & & $124-125$ \\
\hline 33 & BDL33 & 16 & & $124-125$ \\
\hline 34 & BDL34 & & & $124-125$ \\
\hline 35 & BDL35 & & & $124-125$ \\
\hline 36 & BDL36 & 16 & & $124-125$ \\
\hline 37 & BDL37 & $\begin{array}{l}10 \\
157\end{array}$ & & $124-123$ \\
\hline 38 & BDL38 & 16 & & $124-125$ \\
\hline 39 & BDL39 & & & $124-12 J$ \\
\hline 40 & BDL40 & & & $124-125$ \\
\hline 41 & BDL41 & & 3 in 126 & $124-125$ \\
\hline 42 & BDL42 & & 5 iा1 120 & $124-125$ \\
\hline 43 & BDL43 & $16,36,157$ & & $125-126$ \\
\hline 44 & BDL44 & 157 & & $126-127$ \\
\hline 45 & BDL45 & & 3 in 126 & \\
\hline 46 & BDL46 & & 4 in 126 & \\
\hline $\begin{array}{l}40 \\
47\end{array}$ & $\begin{array}{l}\text { BDL47 } \\
\text { BDL }\end{array}$ & & $4 \mathrm{Mll} 120$ & $124-125$ \\
\hline 48 & BDL48 & 16,157 & & $124-125,128-130$ \\
\hline $\begin{array}{l}48 \\
49\end{array}$ & $\begin{array}{l}\text { DDL } 48 \\
\text { not shown }\end{array}$ & $\begin{array}{l}10,157 \\
157\end{array}$ & & $124-125,128-150$ \\
\hline 50 & not shown & 157 & & \\
\hline 51 & not shown & $13,74,157$ & & \\
\hline 52 & not shown & 15,74 & & \\
\hline 53 & HVL1 & & 4 in 124 & \\
\hline 54 & HVL2 & & 4111124 & $121-122$ \\
\hline 55 & HVL3 & & & $122-123$ \\
\hline 56 & HVL4 & & & $122-123$ \\
\hline 57 & HVL5 & & & $118-119$ \\
\hline 58 & HVL6 & & 3 in 124 & \\
\hline 59 & HVL7 & & 1 in 124 & $118-120$ \\
\hline 60 & $\begin{array}{l}\text { HVL } \\
\text { HV } 8\end{array}$ & & 1 in 124 & $110-120$ \\
\hline 61 & HVL9 & & & $107,124,126-128$ \\
\hline $\begin{array}{l}01 \\
62\end{array}$ & $\begin{array}{l}\text { HVL } \\
\text { HVL10 }\end{array}$ & & & $107,124,126-128$ \\
\hline $\begin{array}{l}02 \\
63\end{array}$ & HVL11 & & & $107,124,126-128$ \\
\hline $\begin{array}{l}03 \\
64\end{array}$ & HVL12 & & & 119,124 \\
\hline 65 & HVL13 & & & 124 \\
\hline 66 & HVL14 & 157 & & 124 \\
\hline 67 & HVL15 & & & $122-123$ \\
\hline 68 & not shown & & & \\
\hline 69 & not shown & & & \\
\hline 70 & not shown & & & \\
\hline 71 & not shown & & & \\
\hline 72 & not shown & & & \\
\hline 73 & not shown & & & \\
\hline 74 & not shown & & & \\
\hline 75 & not shown & & & \\
\hline 76 & not shown & & & \\
\hline 77 & not shown & & & \\
\hline 78 & not shown & & & \\
\hline 79 & not shown & & & \\
\hline 80 & not shown & & & \\
\hline 81 & not shown & & & \\
\hline 82 & not shown & & & \\
\hline 83 & not shown & & & \\
\hline
\end{tabular}


Magdalena Czubala et al. / American Journal of Infectious Diseases 8 (3) (2012) 115-122

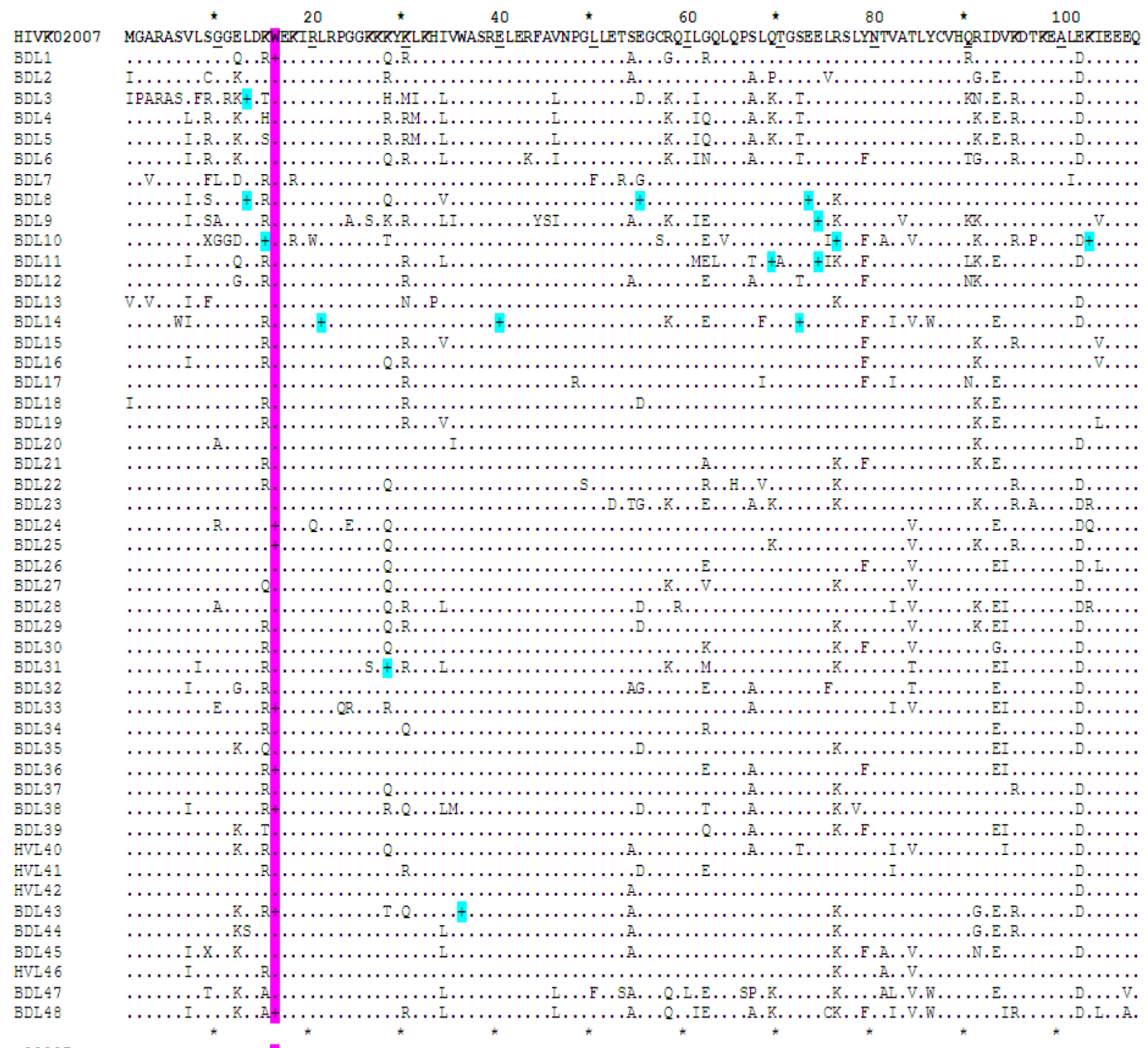

K02007

MGARASVLSGGELDKWEKIRLRPGGKKKYKLKHIVWASRELERFAVNPGLLETSEGCRQILGQLQPSLQTGSEELRSLYNTVATLYCVHQRIDVKDTKEALEKIEEEQ

HVI
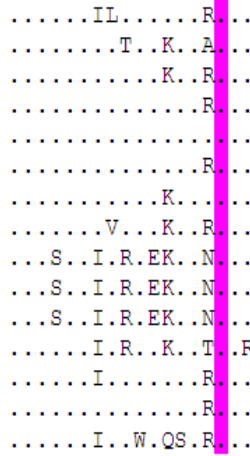

...............

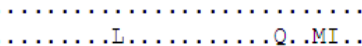


Magdalena Czubala et al. / American Journal of Infectious Diseases 8 (3) (2012) 115-122

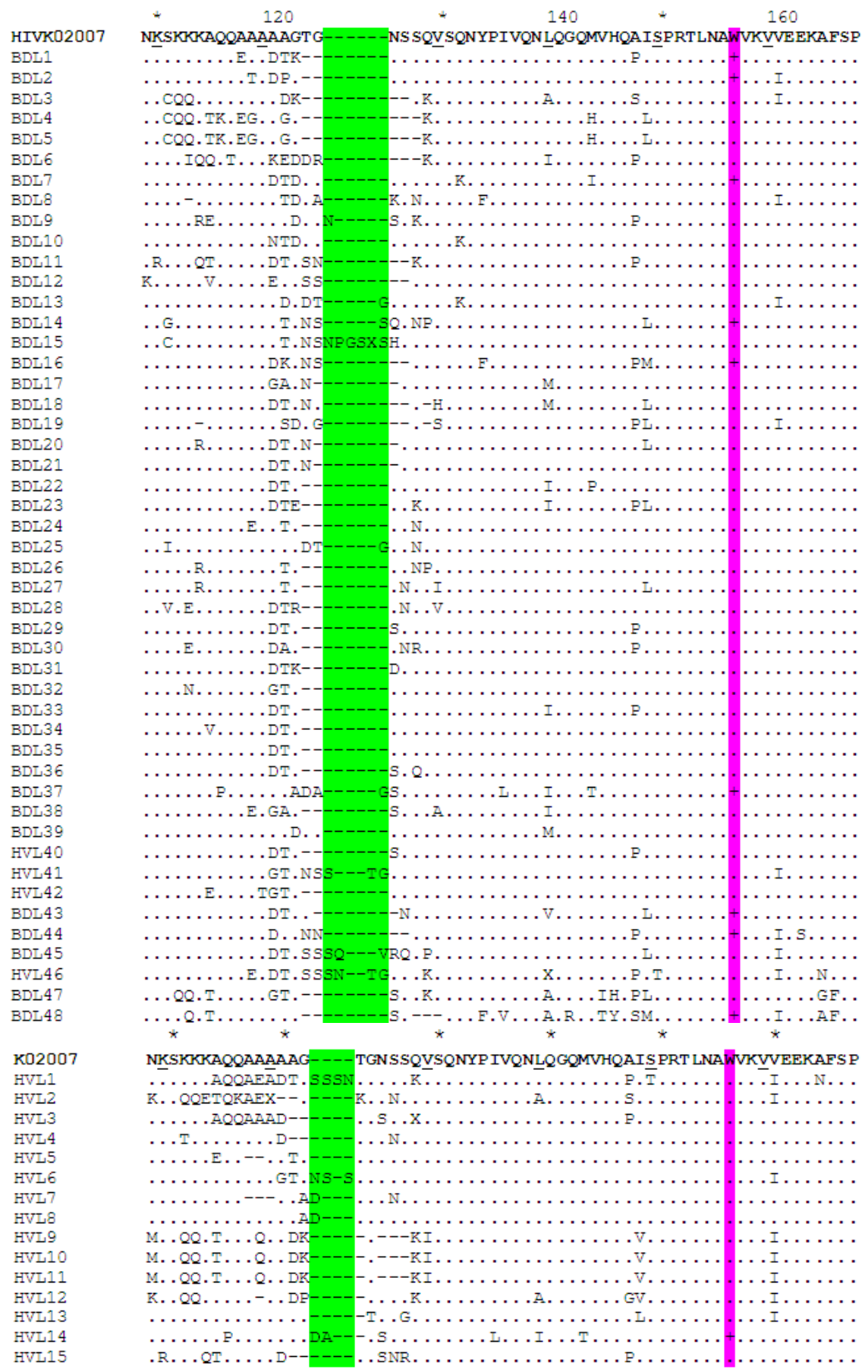

Fig. 1. Alignment of the deduced Gag amino acid from Below Detectable Levels patients (BDL) and patients with detectable plasma viremia (HVL). K02007 sequence serves as HIV-1 Gag reference sequence. Matching amino acids are denoted by dots (.), + stands for stop codons. Pink color indicates positions of $\mathrm{G} \rightarrow$ A hypermutation hotspots 16 and 157 , remaining stop codons are colored turquoise; bright green represents regions with deletions and/or insertions of amino acids into the sequences. 
Magdalena Czubala et al. / American Journal of Infectious Diseases 8 (3) (2012) 115-122

Table 2. Summary showing total number of stop codons between BDL and viremic groups

\begin{tabular}{lcc}
\hline & BDL & Viremic \\
\hline Number of samples & 52.0 & 31.0 \\
Total & 83.0 & \\
Samples with stop codon(s) & 24.0 & 1.0 \\
Percentage (\%) & 46.2 & 5.9 \\
Amino acid numberlstop codon number & & \\
1 to 80 (excl. 16) & 18.0 & 0.0 \\
81 to 168 (excl. 157) & 1.0 & 0.0 \\
16 & 8.0 & 0.0 \\
157 & 12.0 & 1.0 \\
Total number of stop codons & 39.0 & 1.0 \\
p value & & \\
\hline
\end{tabular}

The objective was to determine if mutations other than drug resistance ones, such as single nucleotide changes and/or frame-shift mutations that emerge during HAART, could segregate HIV individuals with or without detectable plasma. Since the majority of antiretroviral drugs target the gag and pol genes of the HIV life cycle, we focused only on mutations that emerge during complete control of plasma viremia with HAART, as they may have the potential to explain how viral fitness and replicative ability is altered transitorily during HAART. We analyzed $83 \mathrm{HIV}+$ individuals by sequencing the 560bp gag gene fragment derived from primary PBMCs of the two HIV+ groups discussed above.

Our data demonstrates that in addition to other pharmacological and virological aspects of drug-mediated control of viremia in HIV+ individuals, viral control was defined by the preponderance of inactivating mutations at position 16 and 157 in the gag gene $(p<0.0001)$ (Fig. 1 and Table 1-2). These comprised of frame interrupting stop codons that were unique to individuals showing BDL of plasma viremia on HAART. These mutations were lost as viremia emerged during therapy, which was apparent from viremic patients, suggesting a possible role in transitory viral inactivation in vivo during HAART. Thus, it is apparent that the gain of these mutations leads to viral control in vivo, whereas the loss of these mutations leads to restoration of viral replication, which is evident from the complete and systematic absence of these mutations at position 16 and 157 of the gag gene derived from viremic patients on HAART.

\section{MATERIALS AND METHODS}

Blood samples from 83 HIV sero-positive individuals were obtained from the Department of
Virology, Centre for Infectious Diseases and Microbiology Laboratory Services, ICPMR, Westmead Hospital and the Australian Red Cross, following informed consent. This study was approved by the Human Ethics Committee of the Sydney West Area Health Service. Prior written consent of patients was obtained for obtaining whole blood. Whole blood (10-20 $\mathrm{mL}$ ) from individuals was collected in heparinised or EDTA vacuum tubes, centrifuged at $800 \mathrm{x}$ g for $10 \mathrm{~min}$ and the cellular fraction collected. This fraction was diluted in sterile PBS define to $35 \mathrm{~mL}$ and carefully layered over $15 \mathrm{~mL}$ of Ficoll Hypaque in a $50 \mathrm{~mL}$ centrifuge tube (Becton Dickinson, Franklin Lakes, NJ, USA). After centrifugation at $800 \mathrm{x}$ g for $20 \mathrm{~min}$, PBMCs were carefully removed from the interface with a transfer pipette and then pelleted by further centrifugation at $400 \mathrm{x} g$ for $10 \mathrm{~min}$. The supernatant was discarded and the pellet resuspended in PBS, then spun again at 400x g for $10 \mathrm{~min}$. DNA extractions were performed using QIAGEN DNeasy Blood + Tissue Kit (Qiagen, Germany), according to the manufacturer's protocol. DNA was visualized on an agarose gel under UV light.

The proviral DNA was amplified using the HIV-specific gag gene primer pairs in a nested Polymerase Chain Reaction (PCR). Double-nested PCRs were used to amplify HIV-1 target templates in all cases and significant care was taken to avoid PCR contamination and carryover problems by conducting PCR in designated and remotely located facility from the DNA laboratories and sterilizing the PCR laboratory with UV irridiation. Negative controls were included in both rounds of amplification in all cases, using material derived from template-free (PBS) DNA extractions. For the gag gene amplification, one primer set was used for external amplification (gag2, MSF12) and a second pair for internal amplification (gag1, gag583R). PCR products were subjected to clean-up prior to sequencing and purified using the 96 -well PCR purification plates on a vacuum manifold. Sequencing was performed using the ABI PRISM BigDye Terminator V3.1 Ready Reaction Cycle Sequencing Kit (Applied Biosystems, CA, USA). Reactions were carried out in a total of $12 \mu \mathrm{L}$ using $1 \mu \mathrm{L}$ of purified PCR product. Unincorporated dye terminators were removed from sequencing reactions by Sephadex purification. For sequence analysis, multiple sequence alignments of the viral sequences were performed using CLUSTALW program (Thompson et al., 1994). Analysis of the frequency of the number of codons differing between BDL and viremic groups was 
performed by Mann-Whitney non-parametric test in the SPSS software package.

\section{RESULTS AND DISCUSSION}

Due to undetectable plasma virus in BDL patients and the limitation it poses in amplifying HIV from plasma of these patients, we took the advantage of HIV-integrated provirus in ex-vivo-derived uncultured PBMCs for DNA amplification. Analysis of the proviral DNA sequences from PBMCs of $83 \mathrm{HIV}+$ individuals on HAART clearly demonstrated that stop codons in the protease gene are prevalent in HIV-1 strains in vivo when plasma HIV load is at below detectable levels $(<20-40$ copies $/ \mathrm{ml}$ plasma). This is in contrast to viremic individuals, where no stop codons were detected $(p<0.0001)$. These observations clearly show an unusual aspect of molecular control of HIV during HAART, which is possibly achieved transitorily via the inactivating mutations at positions 16 and 157 of the protease gene unique to BDL HIV+ patients on HAART. Alternatively, these mutations were also reliable in predicting undetectable plasma viremia in each case. The sequence analyses showed that even though the occurrence of stop codons was a significant feature of HIV+ BDL individuals, both variants (with and without stop codons) prevailed in vivo in most cases, with the higher preponderance of mutant variant at all times in BDL individuals. Although this virus population modulation may be one indication of viral control during HAART, the existence of two populations with and without stop codons in BDL individuals is also suggestive of low-level HIV replication in some cellular reservoirs. Since total PBMCs were analyzed for sequencing, the exact cell type could not be identified. Previously resting memory CD4 $+\mathrm{T}$ cells have been shown to harbor defective viral populations during HAART-induced HIV control (Kieffer et al., 2005). The co-existence of defective and non-defective populations in the blood stream of BDL individuals also suggests that effect of these mutations on viral replication control may be transitory, which we believe is the case. This is further reflected in the comparison against individuals with plasma viremia, where no interrupting stop codons were found and invariably only intact viral genomes were observed. It may also suggest that the absence of stop codons in virus during the viremic phase may signal breakthrough viral population with drug resistance mutations and antiretroviral drug treatment failure, a fact not dissected in this study. Thus, the presence of these inactivating stop codons may harbor some prognostic value.

Of note were the stop codons, which were created via systematic elimination of tryptophan residues (W) by a process of "G-to-A hypermutation" (Pathak and Temin, 1990) in which G-to-A transitions far exceeded all other mutations in viral sequences (Borman et al., 1995). G-to-A hypermutation has been described in about $38 \%$ of asymptomatic individuals and $50 \%$ of seroconverters (Janini et al., 2001), although the functional relevance remains poorly understood. Further, it is unclear whether hypermutation is influenced by viral escape or if it is a function of the cell population harboring the virus. The loss of coding potential of these hypermutated HIV sequences strongly suggests that they are incapable of generating progeny virions and cannot contribute to the HIV gene pool (Janini et al., 2001). Consistent with these findings, we have previously reported a case of a long-term non-progressor where G-to-A hypermutation was significantly higher in the gag and pol genes, some of which resulted in stop codons (Wang et al., 2003). It is believed that the PBMC-derived proviral sequences may display a higher incidence of G-A hypermutation (Janini et al., 2001). However, a recent longitudinal analysis of full-length HIV-1 genomes from an American cohort of 20 slow progressors and non-progressors (Wang et al., 2000) did not show a predominance of G-A hypermutation in the non-progressors, thereby suggesting a minimal role of this phenomenon in defining the rate of disease progression. Nonetheless, from our analyses it appears that the G-A changes resulting in stop codons in the protease gene correlate with low or non-replicative virus. This comparative observation that HIV strains from viremic individuals being completely devoid of stop codons suggests that GA hypermutation may play a significant role in rendering HIV genomes defective and may be important in containing HIV disease progression in some cases. Nonetheless, the comparison of BDL and viremic patients clearly show that this is a transitory phenomenon and further investigations are needed to dissect the exact relevance of these mutations in the context of HIV disease progression. HIV culture studies have previously shown that G-A hypermutation may be transitory and can be induced by mitogens (Janini et al., 2001). This in vitro situation appears to be consistent with the in vivo situation we have observed in our study. But the only difference is that these G-A changes are taking place in HIV patients while on HAART. Overall, supporting our 
observations, Kieffer et al. (2005) also showed interrupting protease gene stop codons in the gag region in 9/9 individuals from the resting memory CD4+ T cells in individuals with below detectable levels of plasma HIV. This transitory control of plasma HIV to BDL during HAART may provide insights into a more durable HIV control once the biological role and mechanism of the emergence of these novel mutations is more clearly understood.

\section{CONCLUSION}

Overall, these analyses are the first to point out that the occurrence of transitory stop codons in HIV strains during HAART are able to sustain below detectable levels of HIV for a certain period of time and there is quasispecies modulation in vivo between mutant and wild-type populations within the gag gene, which eventually defines the predominant variant in vivo. Our data suggest that HAART is able to contain HIV in plasma to below detectable levels by impairing the ability of HIV to replicate due to stop codons, we have reported herein. It should be emphasized that HIV protease can tolerate substantial amount of mutations; at least one third of its 99 amino acids can deviate from the wild-type sequence without altering function, but the mutations at positions 16 (aa residue 5) and 157 (aa residue 52) as noted in this study are unique and have not been reported before. In addition, these mutations lie outside active-site triad (Asp25, Thr26, Gly27) of HIV protease, which is located in a loop whose structure is stabilized by a network of hydrogen bonds similar to that in the eukaryotic enzymes (Wlodawer and Erickson, 1993). Further functional studies are needed to clarify the role of these mutations in transitory viral replication control in vivo and also possibly in predicting fluctuating plasma concentrations of the drugs and emergence of drug resistance.

\section{ACKNOWLEDGMENTS}

NKS, BW and DED were funded by the National Center for HIV and Hepatitis for this study. MC was the recipient of the James Rennie Bequest from the University of Edinburgh, UK. BW is the recipient of the NHMRC RD Wright fellowship.

\section{REFERENCES}

Borman, A.M., C. Quillent, P. Charneau, K.M. Kean and F. Clavel, 1995. A highly defective HIV-1 Group O provirus: Evidence for the role of local sequence determinants in $\mathrm{G} \rightarrow \mathrm{A}$ hypermutation during negative-strand viral DNA synthesis. Virology, 208: 601-609. DOI: 10.1006/viro.1995.1191

Janini, M., M. Rogers, D.R. Birx and F.E. McCutchan, 2001. Human immunodeficiency virus type 1 DNA sequences genetically damaged by hypermutation are often abundant in patient peripheral blood mononuclear cells and may be generated during near-simultaneous infection and activation of $\mathrm{CD} 4{ }^{+}$ $\mathrm{T}$ cells. J. Virol., 75: 7973-7986. DOI: 10.1128/JVI.75.17.7973-7986.2001

Kieffer, T.L., P. Kwon, R.E. Nettles, Y. Han and S.C. Ray et al., 2005. G $\rightarrow$ A hypermutation in protease and reverse transcriptase regions of human immunodeficiency virus type 1 residing in resting $\mathrm{CD}^{+}$T cells in vivo. J. Virol., 79: 1975-1980. DOI: 10.1128/JVI.79.3.1975-1980.2005

Pathak, V.K. and H.M. Temin, 1990. Broad spectrum of in vivo forward mutations, hypermutations and mutational hotspots in a retroviral shuttle vector after a single replication cycle: Substitutions, frameshifts and hypermutations. Proc. Natl. Acad. Sci. USA., 87: 6019-6023. PMID: 2201018

Potter, S.J., D.E. Dwyer and N.K. Saksena, 2003. Differential cellular distribution of HIV-1 drug resistance in vivo: Evidence for infection of CD8+ T cells during HAART. Virology, 305: 339-352. DOI: 10.1006/viro.2002.1703

Potter, S.J., P. Lemey, G. Achaz, C.B. Chew and A.M. Vandamme et al., 2004. HIV-1 compartmentalization in diverse leukocyte populations during antiretroviral therapy. J. Leu. Biol., 76: 562-570. DOI: 10.1189/jlb.0404234

Potter, S.J., P. Lemey, W.B. Dyer, J.S. Sullivan and C.B. Chew et al., 2006. Genetic analyses reveal structured HIV-1 populations in serially sampled T lymphocytes of patients receiving HAART. Virology, 348: 35-46. $\quad$ DOI: 10.1016/j.virol.2005.12.031

Thompson, J.D., D.G. Higgins and T.J. Gibson, 1994. CLUSTAL W: Improving the sensitivity of progressive multiple sequence alignment through sequence weighting, position-specific gap penalties and weight matrix choice. Nucl. Acid Res., 22: 4673-4680. DOI: 10.1093/nar/22.22.4673 
Wang, B., M. Mikhail, W.B. Dyer, J.J. Zaunders and A.D. Kelleher et al., 2003. First demonstration of a lack of viral sequence evolution in a nonprogressor, defining replication-incompetent HIV-1 infection. Virology, 312: 135-150. DOI: 10.1016/S00426822(03)00159-4

Wang, B., T.J. Spira, S. Owen, R.B. Lal and N.K. Saksena, 2000. HIV-1 strains from a cohort of American subjects reveal the presence of a V2 region extension unique to slow progressors and non-progressors. AIDS, 14: 213-223. PMID: 10716496
Wlodawer, A. and J.W. Erickson, 1993. Structure-based inhibitors of HIV-1 protease. Annu. Rev. Biochem., 62: 543-585.

DOI: 